\title{
EXPLICIT BOUNDS ON THE LOGARITHMIC DERIVATIVE AND THE RECIPROCAL OF THE RIEMANN ZETA-FUNCTION
}

\author{
TIM TRUdGIAN
}

Abstract: The purpose of this article is consider $\left|\zeta^{\prime}(\sigma+i t) / \zeta(\sigma+i t)\right|$ and $|\zeta(\sigma+i t)|^{-1}$ when $\sigma$ is close to unity. We prove that $\left|\zeta^{\prime}(\sigma+i t) / \zeta(\sigma+i t)\right| \leqslant 87 \log t$ and $|\zeta(\sigma+i t)|^{-1} \leqslant 6.9 \times 10^{6} \log t$ for $\sigma \geqslant 1-1 /(8 \log t)$ and $t \geqslant 45$.

Keywords: Riemann zeta-function, prime number theorem, zero-free region.

\section{Introduction}

Consider $\mu(n)$ the Möbius function, $M(x)=\sum_{n \leqslant x} \mu(n)$ and $m(x)=\sum_{n \leqslant x} \mu(n) / n$. It is known that $M(x) / x$ and $m(x)$ both tend to zero as $x$ tends to infinity. Schoenfeld [10] showed that $|M(x)| / x \leqslant 2.9 /(\log x)$ for $x>1$; this was improved by Ramaré [9] who showed that $|M(x)| / x \leqslant 0.013 /(\log x)$ for $x \geqslant 1.1 \times 10^{6}$. Ramaré [op. cit.] also proved that $|m(x)| \leqslant 0.026 /(\log x)$ for $x \geqslant 61000$.

One can produce explicit bounds of the form

$$
|m(x)| \leqslant C_{1} \log ^{3} x \exp \left(-C_{2} \sqrt{\log x}\right),
$$

where $C_{1}, C_{2}>0$, by following the arguments in $\S 3.13$ in [13]. Indeed, since $\sum_{n=1}^{\infty} \mu(n) / n^{s}=\zeta(s)^{-1}$ for all $\Re s=\sigma>1$, one can use Perron's formula to show that

$$
\sum_{n<x} \frac{\mu(n)}{n^{1+i t}}=\frac{1}{2 \pi i} \int_{c-i T}^{c+i T} \frac{1}{\zeta(1+i t+w)} \frac{x^{w}}{w} d w+E(c, x, T),
$$

where $c>0$ and $E(c, x, T)$ is an error term that can be estimated explicitly. If one has an explicit zero-free region for $\zeta(s)$, and an explicit bound for $|\zeta(s)|^{-1}$ in $\sigma \geqslant 1-1 /(W \log t)$, then one may apply Cauchy's theorem to the integral in (1.2) and prove (1.1) with $C_{2}=1 / W$. One can recover explicit bounds for $M(x)$ using (1.1) and partial summation. 
Similarly, if one has a bound for $\left|\zeta^{\prime}(s) / \zeta(s)\right|$ one may follow $\S 3.14$ in [13] to bound $\psi(x)=\sum_{n \leqslant x} \Lambda(n)$, where $\Lambda(n)$ is the von Mangoldt function. Finally, one can consider $L(x) \stackrel{=}{=} \sum_{n<x} \lambda(n)$, where $\lambda(n)$ is Liouville's function, which defines the Dirichlet series $\zeta(2 s) / \zeta(s)=\sum_{n=1}^{\infty} \lambda(n) n^{-s}$, for $\sigma>1$. Provided that we have an explicit bound for $|\zeta(s)|^{-1}$, we may apply Perron's formula to obtain an explicit bound for $L(x)$.

Given the applications to $M(x), m(x), \psi(x)$ and $L(x)$, it seems natural to try to obtain an explicit bound for $|\zeta(s)|^{-1}$ and for $\left|\zeta^{\prime}(s) / \zeta(s)\right|$. The point of this article is to prove

Theorem 1. For $t \geqslant 45$ and for $\sigma \geqslant 1-1 /(8 \log t)$ we have

$$
\left|\frac{\zeta^{\prime}(s)}{\zeta(s)}\right| \leqslant 87 \log t, \quad \frac{1}{|\zeta(s)|} \leqslant 6.9 \times 10^{6} \log t .
$$

Moreover, for $s$ in the region $t \geqslant t_{0}$ and $\sigma \geqslant 1-1 /(W \log t)$, bounds of the sort $\left|\zeta^{\prime}(s) / \zeta(s)\right| \leqslant R_{1} \log t$ and $|\zeta(s)|^{-1} \leqslant R_{2} \log t$ are given in Table 1 .

The method of proof follows that in Titchmarsh [13, pp. 56-60]. In $§ 2$ explicit versions of Titchmarsh's Lemmas $\alpha$ and $\gamma$ are given. These were first annunciated by Landau [5]. Bounds similar to those in (1.3), but without explicit constants, were proved by Gronwall [2, p. 96].

Landau's method contains two steps. First, one uses good bounds for $\zeta(s)$ near $\sigma=1$ to deduce a zero-free region near $\sigma=1$. Second, the bound on $\zeta(s)$ and the zero-free region are used to bound $\left|\zeta^{\prime}(s) / \zeta(s)\right|$ and $|\zeta(s)|^{-1}$. We break into this argument after the first step. Instead of using the zero-free region obtained by Landau's method we use the one obtained by Kadiri [4]. This sharper zero-free region enables us to obtain relatively good bounds on $\left|\zeta^{\prime}(s) / \zeta(s)\right|$ and $|\zeta(s)|^{-1}$.

It should be remarked that Ford's [1] theorem, that

$$
|\zeta(\sigma+i t)| \leqslant 76.2 t^{4.45(1-\sigma)^{3 / 2}} \log ^{2 / 3} t, \quad\left(t \geqslant 3, \frac{1}{2} \leqslant \sigma \leqslant 1\right),
$$

could be used to obtain results of the form

$$
\frac{1}{|\zeta(s)|} \leqslant A(\log t)^{2 / 3}(\log \log t)^{1 / 3}
$$

for some constant $A$, as well as a similar result for $\left|\zeta^{\prime}(s) / \zeta(s)\right|$. One could burn the extra candle and estimate the size of the constant $A$. However it is likely that such results would improve on those in Theorem 1 only when $t$ is extremely large.

\section{Acknowledgements}

I am grateful to Olivier Ramare for suggesting the problem to me, and for the referee for bringing Gronwall's result to my attention. 


\section{Preparatory lemmas}

Lemma 1. Let $f(s)$ be regular and let $\left|\frac{f(s)}{f\left(s_{0}\right)}\right| \leqslant A_{1}$ in $\left|s-s_{0}\right| \leqslant r$. Then

$$
\left|\frac{f^{\prime}(s)}{f(s)}-\sum_{\rho} \frac{1}{s-\rho}\right| \leqslant \frac{4 \log A_{1}}{r(1-2 \alpha)^{2}}, \quad\left(\left|s-s_{0}\right| \leqslant \alpha r\right)
$$

where $\rho$ runs through the zeroes of $f(s)$ for which $|s-\rho| \leqslant \frac{1}{2} r$, and where $\alpha<\frac{1}{2}$.

Proof. See [11, p. 151].

Whereas Titchmarsh [13, Lemma $\alpha]$ proves Lemma 1 by applying the BorelCarathéodory theorem and then Cauchy's theorem for derivatives, Tenenbaum in [11] proves Lemma 1 'in one go'. This diminishes the right hand side of (2.1). For example, when $\alpha=\frac{1}{4}$ the proof in [13] gives $48 \log A_{1} / r$, whereas Lemma 1 gives $16 \log A_{1} / r$.

Lemma 2. Let $f(s)$ satisfy the conditions of Lemma 1, and let $\left|\frac{f^{\prime}\left(s_{0}\right)}{f\left(s_{0}\right)}\right| \leqslant \frac{A_{2}}{r}$. Suppose also that $f(s) \neq 0$ when $\left|s-s_{0}\right| \leqslant r$ and $\sigma \geqslant \sigma_{0}-\eta r^{\prime}$, where $\eta>1$ and $\eta r^{\prime} \leqslant \alpha r$. Then

$$
\left|\frac{f^{\prime}(s)}{f(s)}\right| \leqslant \frac{8 \alpha \log A_{1}}{r(\eta-1)(1-2 \alpha)^{2}}+\frac{\eta+1}{\eta-1} \frac{A_{2}}{r}, \quad\left(\left|s-s_{0}\right| \leqslant r^{\prime}\right) .
$$

Proof. In the region $\left|s-s_{0}\right| \leqslant \alpha r$, bound the real part of $f^{\prime}(s) / f(s)$ using Lemma 1 and note that, for $\sigma \geqslant \sigma_{0}-\eta r^{\prime}$, we have $\Re(s-\rho)>0$. Now apply the Borel-Carathéodory theorem (see, e.g., $[12, \S 5.5])$ to the function $-f^{\prime}(s) / f(s)$ on the circles $\left|s-s_{0}\right|=\eta r^{\prime}$ and $\left|s-s_{0}\right|=r^{\prime}$.

We shall also require the following bound on $\zeta(s)$ which we shall borrow from [14].

Lemma 3 (Cor. 1 [14]). Let $\delta$ be a positive real number and let

$$
a_{0}\left(\sigma, Q_{0}, t\right)=\frac{\sigma+Q_{0}}{2 t^{2} \log t}+\frac{\pi}{2 \log t}+\frac{\pi\left(\sigma+Q_{0}\right)^{2}}{4 t \log ^{2} t}, \quad a_{1}\left(\sigma, Q_{0}, t\right)=\frac{\sigma+Q_{0}}{t}
$$

Then, for $\sigma \in\left[\frac{1}{2}, 1+\delta\right]$ and $t \geqslant t_{0}$ we have

$$
|\zeta(s)| \leqslant 0.732\left(1+a_{1}\left(1+\delta, 5, t_{0}\right)\right)^{7 / 6}\left(1+a_{0}\left(1+\delta, 5, t_{0}\right)\right)^{2} t^{1 / 6} \log t,
$$

provided that

$$
t \geqslant \max \{1.16, \exp [4 \zeta(1+\delta) / 3]\}
$$




\section{Estimating $\left|\zeta^{\prime}(s) / \zeta(s)\right|$}

First consider $t_{0} \geqslant H$, where $H=3.06 \times 10^{10}$ is the height to which the Riemann hypothesis has been verified - see [7]. Let $s_{0}=\sigma_{0}+i t_{0}=1+\frac{c}{\log t_{0}}+i t_{0}$, where $c$ is a positive constant to be determined later. We aim at applying Lemma 2 with $r=\frac{1}{2}$. In the region $\left|s-s_{0}\right| \leqslant \frac{1}{2}$ we have

$$
\frac{1}{2} \leqslant \sigma \leqslant 1+\frac{1}{2}+\frac{c}{\log H}, \quad t \leqslant t_{0}\left(1+\frac{1}{2 H}\right) .
$$

We shall apply Lemma 3 with $\delta=\frac{1}{2}+\frac{c}{\log H}$; the condition in (2.3) is certainly met for all $t \geqslant 34$. This shows that

$$
|\zeta(s)| \leqslant 0.732 \alpha_{1} t_{0}^{\frac{1}{6}} \log t_{0}, \quad\left(\left|s-s_{0}\right| \leqslant \frac{1}{2}\right),
$$

where

$$
\alpha_{1}=\left(1+a_{1}\left(\frac{3}{2}+\frac{c}{\log H}, 5, H-\frac{1}{2}\right)\right)^{\frac{7}{6}}\left(1+a_{0}\left(\frac{3}{2}+\frac{c}{\log H}, 5, H-\frac{1}{2}\right)\right)^{2}\left(1+\frac{1}{2 H}\right)^{\frac{7}{6}} .
$$

We now bound $\left|\zeta\left(s_{0}\right)\right|$ trivially using the estimate $\left|\zeta\left(s_{0}\right)\right| \geqslant \zeta\left(2 \sigma_{0}\right) / \zeta\left(\sigma_{0}\right)$. This, together with (3.1), shows that

$$
\left|\frac{\zeta(s)}{\zeta\left(s_{0}\right)}\right| \leqslant A_{1}:=0.732 \alpha_{1} t_{0}^{\frac{1}{6}}\left(\log t_{0}\right)^{2} \frac{X\left(1+\frac{c}{\log H}\right)}{c}
$$

where

$$
X(t)=\frac{\zeta(t)(t-1)}{\zeta(2 t)} .
$$

Note that $X(t)$ is increasing and that $\lim _{t \rightarrow 1} X(t)=6 \pi^{-2}$.

To bound $\left|\zeta^{\prime}(s) / \zeta(s)\right|$ we use the trivial bound $\left|\zeta^{\prime}(s) / \zeta(s)\right| \leqslant-\zeta^{\prime}(\sigma) / \zeta(\sigma)$ and Lemma 70.1 in [3], which shows that $-\zeta^{\prime}(x) / \zeta(x)<1 /(x-1)$ for any real $x>1$. We therefore have

$$
\left|\frac{\zeta^{\prime}\left(s_{0}\right)}{\zeta\left(s_{0}\right)}\right| \leqslant \frac{A_{2}}{r}, \quad \text { where } A_{2}=\frac{r \log t_{0}}{c}, \quad r=\frac{1}{2} .
$$

\subsection{Using the zero-free region}

Suppose

$$
\zeta(s) \neq 0, \quad \text { for } \quad \sigma \geqslant 1-\frac{1}{R \log t}, \quad(t \geqslant 3) .
$$

Kadiri [4] has shown that one may take $R=5.69693$. We keep the parameter $R$ in the equations that follow. Let $t^{\prime}$ be a real number for which

$$
\frac{c}{\log t_{0}}+\frac{1}{R \log \left(t_{0}+t^{\prime}\right)}<t^{\prime}
$$


It follows that there are no zeroes of $\zeta(s)$ in the region $\left|s-s_{0}\right| \leqslant 1+\frac{c}{\log t_{0}}-$ $\frac{1}{R \log \left(t_{0}+t^{\prime}\right)}$. We may convert this into a slightly easier form to show that there are no zeroes of $\zeta(s)$ in the region

$$
\left|s-s_{0}\right| \leqslant \frac{c+\frac{1}{\alpha_{2} R}}{\log t_{0}}
$$

where

$$
\alpha_{2}=1+\frac{t^{\prime}}{H \log H} .
$$

To apply Lemma 2 we choose

$$
\eta r^{\prime}=\alpha r=\frac{1}{2} \alpha=\frac{c+\frac{1}{\alpha_{2} R}}{\log t_{0}}
$$

whence

$$
r^{\prime}=\frac{c+\frac{1}{\alpha_{2} R}}{\eta \log t_{0}}, \quad \alpha \leqslant \frac{2\left(c+\frac{1}{\alpha_{2} R}\right)}{\log H} .
$$

We use Lemma 2 and (3.2), (3.3), and (3.4) to prove

Lemma 4. For $t_{0} \geqslant H$

$$
\left|\frac{\zeta^{\prime}(s)}{\zeta(s)}\right| \leqslant A \log t_{0}+B \log \log t_{0}+C, \quad\left(\left|s-s_{0}\right| \leqslant \frac{c+\frac{1}{\alpha_{2} R}}{\eta \log t_{0}}\right),
$$

where

$$
\begin{aligned}
A & =\frac{8}{3(\eta-1)(1-2 \alpha)^{2}}+\left(\frac{\eta+1}{\eta-1}\right) \frac{1}{2 c}, \\
B & =\frac{32}{(\eta-1)(1-2 \alpha)^{2}}, \\
C & =\frac{16\left(\log \alpha_{1}+\log (0.732 X / c)\right)}{(\eta-1)(1-2 \alpha)^{2}} .
\end{aligned}
$$

The bound in (3.5) holds whenever

$$
\sigma_{0}-\frac{c+\frac{1}{\alpha_{2} R}}{\eta \log t_{0}} \leqslant \sigma \leqslant \sigma_{0}+\frac{c+\frac{1}{\alpha_{2} R}}{\eta \log t_{0}} .
$$

For larger values of $\sigma_{0}$ we use the trivial bound on $\left|\zeta^{\prime}(s) / \zeta(s)\right|$. Making the substitution $t_{0} \mapsto t$ we obtain a bound on $\left|\zeta^{\prime}(s) / \zeta(s)\right|$ for all $\sigma>1-1 /(W \log t)$ for some constant $W$. The result is summarised in

Theorem 2. Let

$$
W=\frac{\eta \alpha_{2} R}{1+(1-\eta) \alpha_{2} R c}, \quad\left(\alpha_{2} R c(\eta-1)<1\right) .
$$


Then, for all $t \geqslant H$ and for $\sigma \geqslant 1-1 /(W \log t)$ we have

$$
\left|\frac{\zeta^{\prime}(\sigma+i t)}{\zeta(\sigma+i t)}\right| \leqslant R_{1} \log t
$$

where

$$
R_{1}=\max \left\{\frac{\eta}{\eta c+c+\frac{1}{\alpha_{2} R}}, A+B \frac{\log \log H}{\log H}+\frac{C}{\log H}\right\} .
$$

For a given $W$, we solve (3.6) for $\eta$ and evaluate $R_{1}$ in (3.7) by varying $c \in\left[10^{-4}, 1\right]$ in increments of $10^{-4}$. For example, when $R=5.69693$, given $W=8$, choosing $c=0.1369$ gives $R_{1} \leqslant 86.23$.

We now turn to the case when $0<T_{0}<t<H$. In this case there are no zeroes for $\sigma_{0}-\eta r^{\prime}>\frac{1}{2}$. We therefore choose

$$
\eta r^{\prime}=\alpha r=\frac{1}{2} \alpha, \quad \text { whence } r^{\prime}=\frac{\alpha}{2 \eta}
$$

where we require that $\alpha$ be less than $\frac{1}{2}$ to ensure that the conditions of Lemma 1 are satisfied. We now follow the argument leading to Lemma 4 , noting the change of $\alpha$ and of $r^{\prime}$. We arrive at a bound for $\left|\zeta^{\prime}(s) / \zeta(s)\right|$ in the region $\sigma \geqslant \sigma_{0}-\alpha /(2 \eta)$. This region will be at least as wide as that in Theorem 2 if

$$
\frac{\frac{\alpha}{2} \log T_{0}-c \eta}{\eta} \geqslant \frac{1}{W}
$$

We use (3.8) to solve for $T_{0}$. We then optimise by varying $\alpha \in\left[10^{-2}, 1\right]$ in increments of $10^{-2}, \eta \in[1.001,3]$ in increments of $10^{-3}$, and $c \in[0.001,1]$ in increments of $10^{-3}$. We compare the value of $R_{1}$ thus obtained with that obtained when $t \geqslant H$. For example, when $W=8$ we have already shown that $R_{1} \leqslant 86.23$ for all $t \geqslant H$. Choosing $\alpha=0.23, c=0.041, \eta=2.631$ we have $R_{1} \leqslant 86.11$ with $W=8$ and $t \geqslant 44.61$. We continue in this way for other values of $W$ : the results on $R_{1}$ are presented in Table 1.

\section{Bounding $1 /|\zeta(s)|$}

We follow the argument on page 60 of [13]. If $1-\frac{1}{W \log t} \leqslant \sigma \leqslant 1+\frac{d}{\log t}$, for some $d>0$, then, by Theorem 2 , we have

$$
\begin{aligned}
\log \frac{1}{|\zeta(s)|} & \leqslant-\Re \log \zeta(s) \\
& =-\Re \log \zeta\left(1+\frac{d}{\log t}+i t\right)+\int_{\sigma}^{1+\frac{d}{\log t}} \Re \frac{\zeta^{\prime}}{\zeta}(\xi+i t) d \xi \\
& \leqslant \log \zeta\left(1+\frac{d}{\log t}\right)-\log \zeta\left(2\left(1+\frac{d}{\log t}\right)\right)+R_{1}\left(d+\frac{1}{W}\right)
\end{aligned}
$$


for $t \geqslant t_{0}$ where $t_{0}, W$ and $R_{1}$ are in Table 1 . Write

$$
\zeta(\sigma)=\zeta(\sigma)(\sigma-1) /(\sigma-1)=Y(\sigma) /(\sigma-1),
$$

whence

$$
|\zeta(s)|^{-1} \leqslant \frac{Y\left(1+\frac{d}{\log t_{0}}\right) e^{R_{1}(d+1 / W)}}{d \zeta\left(2\left(1+\frac{d}{\log t_{0}}\right)\right)} \log t, \quad(1-1 /(W \log t) \leqslant \sigma \leqslant 1+d / \log t) .
$$

If $\sigma_{1} \geqslant \sigma \geqslant 1+\frac{d}{\log t}$ we have

$$
|\zeta(s)|^{-1} \leqslant \frac{X\left(\sigma_{1}\right)}{d} \log t
$$

Finally, for $\sigma \geqslant \sigma_{1}$ we have

$$
|\zeta(s)|^{-1} \leqslant \frac{\zeta\left(\sigma_{1}\right)}{\zeta\left(2 \sigma_{1}\right)} \leqslant \frac{\zeta\left(\sigma_{1}\right)}{\zeta\left(2 \sigma_{1}\right) \log t_{0}} \log t .
$$

We now optimise the maximum of (4.1), (4.2) and (4.3) by varying $d \in\left[10^{-4}, 1\right)$ in increments of $10^{-4}$. The values of $R_{2}$ are presented in Table 1: this proves Theorem 1.

Table 1: Bounds for $\left|\zeta^{\prime}(s) / \zeta(s)\right| \leqslant R_{1} \log t$ and $|\zeta(s)|^{-1} \leqslant R_{2} \log t$ and in $\sigma \geqslant 1-1 /(W \log t)$ for $t \geqslant t_{0}$

\begin{tabular}{|r|r|c|c|}
\hline$W$ & $R_{1}$ & $R_{2}$ & $t_{0}$ \\
\hline 6 & 548.53 & $7.8 \times 10^{43}$ & 34 \\
7 & 140.03 & $1.3 \times 10^{11}$ & 34 \\
8 & 86.23 & $6.9 \times 10^{6}$ & 44.61 \\
9 & 64.98 & $1.5 \times 10^{5}$ & 63.91 \\
10 & 53.60 & $1.9 \times 10^{4}$ & 79.35 \\
11 & 46.50 & $5.3 \times 10^{3}$ & 95.45 \\
12 & 41.64 & 2252 & 113.30 \\
\hline
\end{tabular}

\section{Conclusion}

The dominant factor in (4.1) is $d^{-1} \exp \left(R_{1}(d+1 / W)\right)$. It is the exponential dependence on $R_{1}$ that leads to such large values of $R_{2}$ in Table 1 . Both $R_{1}$ and $R_{2}$ would be diminished were one in possession of any of the following: a higher height to which the Riemann hypothesis has been proved (a larger value of $H$ ), a wider zero-free region (a smaller value of $R$ ), or a better bound on $\zeta(s)$ across the critical strip (improving (2.2)). As noted in [8], the bound in (2.2) appears to be far from optimal. It is hoped that future researchers are able to improve on the methods of attacking this problem. 


\subsection{Note added in proof}

Recently, in [6] it was announced that one could take $R=5.573412$. Conditional on this bound of $R$ one could refine the bounds in Table 1 as follows.

Table 2: Bounds for $\left|\zeta^{\prime}(s) / \zeta(s)\right| \leqslant R_{1} \log t$ and $|\zeta(s)|^{-1} \leqslant R_{2} \log t$ and in $\sigma \geqslant 1-1 /(W \log t)$ for $t \geqslant t_{0}$ - with $R=5.573412$

\begin{tabular}{|r|c|c|c|}
\hline$W$ & $R_{1}$ & $R_{2}$ & $t_{0}$ \\
\hline 6 & 382.58 & $3.2 \times 10^{30}$ & 34 \\
7 & 125.60 & $1.3 \times 10^{10}$ & 34 \\
8 & 80.38 & $3.1 \times 10^{6}$ & 50.28 \\
9 & 61.54 & $9.6 \times 10^{4}$ & 70.59 \\
10 & 51.19 & $1.5 \times 10^{4}$ & 90.87 \\
11 & 44.65 & $4.4 \times 10^{3}$ & 111.12 \\
12 & 40.14 & 1900 & 132.16 \\
\hline
\end{tabular}

\section{References}

[1] K. Ford, Vinogradov's integral and bounds for the Riemann zeta function, Proc. London Math. Soc. 85(3) (2002), 565-633.

[2] T.H. Gronwall, Sur la fonction $\zeta(s)$ de Riemann au voisinage de $\sigma=1$, Palermo Rend. 35(1) (1913), 95-102.

[3] R.R. Hall and G. Tenenbaum, Divisors, vol. 90 of Cambridge Tracts in Math., Cambridge University Press, Cambridge, 1988.

[4] H. Kadiri, Une région explicite sans zéros pour la fonction $\zeta$ de Riemann, Acta Arith. 117(4) (2005), 303-339.

[5] E. Landau, Über die Wurzeln der Zetafunktion, Math. Z. 20 (1924), 98-104.

[6] M.J. Mossinghoff and T.S. Trudgian, Nonnegative trigonometric polynomials and a zero-free region for the Riemann zeta-function, submitted, 2014, preprint available at arXiv:1410.3926 [math.NT].

[7] D.J. Platt, Computing $\pi(x)$ analytically, Math. Comp. 84(293) (2015), 15211535.

[8] D.J. Platt and T.S. Trudgian, An improved explicit bound on $|\zeta(1 / 2+i t)|$, J. Number Theory 147 (2015), 842-851.

[9] O. Ramaré, From explicit estimates for primes to explicit estimates for the Möbius function, Acta Arith. 157(4) (2013), 365-379

[10] L. Schoenfeld, An improved estimate for the summatory function of the Möbius function, Acta Arith. 15 (1969), 221-233.

[11] G. Tenenbaum, Introduction to Analytic and Probabilistic Number Theory, vol. 46 of Cambridge Studies in Advanced Mathematics, Cambridge University Press, Cambridge, 1995. 
[12] E.C. Titchmarsh, The Theory of Functions, Oxford Science Publications, Oxford University Press, Oxford, 2nd edition, 1932.

[13] E.C. Titchmarsh, The Theory of the Riemann zeta-function, Oxford Science Publications, Oxford University Press, Oxford, 2nd edition, 1986.

[14] T.S. Trudgian, Improvements to Turing's method II, Rocky Mountain J. Math., to appear.

Address: Tim Trudgian: Mathematical Sciences Institute, The Australian National University, ACT 0200, Australia.

E-mail: timothy.trudgian@anu.edu.au

Received: 22 April 2014; revised: 13 January 2015 